\title{
Mise au point d'un élevage permanent de Diprion pini L. (Hym. diprionidae) en laboratoire
}

\author{
F. Goussard et C. Geri \\ INRA, station de zoologie forestière, Ardon 45160 Olivet, France
}

(reçu le 25 mars 1989; accepté le 19 juin 1989)

Résumé - Un élevage permanent de Diprion pini L. est obtenu en laboratoire à partir d'insectes provenant de Sologne et de la forêt de Rambouillet au sud de Paris. Les insectes ont été élevés depuis plus de 10 générations en ne reproduisant à chaque génération que des individus non diapausants et en maintenant les larves dans des conditions de photopériode et de température propres à éviter la diapause.

Les larves sont soumises à des températures et à des photophases croissantes : $16^{\circ} \mathrm{C}, 15 \mathrm{~h}$ d'éclairement journalier jusqu'au $3^{\ominus}$ stade, $20^{\circ} \mathrm{C}, 16 \mathrm{~h} 30$ d'éclairement ensuite. La ponte et le développement des larves jusqu'au $3^{\ominus}$ stade s'effectuent sur un pin sylvestre sans manipulation : les larves sont ensuite élevées sur des rameaux coupés renouvelés tous les 2-3 j. Différentes enceintes et 2 types d'éclairage fluorescents sont utilisés.

Des fluctuations importantes du taux de diapause sont observées de génération en génération, sans que leur liaison avec un processus biologique propre à l'insecte ou avec les conditions d'élevage, puissent être clairement établie. II apparaît toutefois que les modifications du type d'éclairage causent d'importantes variations du pourcentage d'individus diapausants. En particulier, les tubes fluorescents utrue-lite» dont le spectre est très proche de celui de la lumière solaire, et qui permettent, par ailleurs, une bonne réalisation de l'accouplement et de la ponte, paraissent accroître le nombre d'insectes en diapause. Dans tous les cas, on obtient des taux importants de sorties immédiates des adultes après un certain nombre de générations.

Un accroissement important de la sex-ratio est observé, dans l'élevage, qui peut avoir plusieurs causes, notamment la consanguinité.

L'élevage, tel qu'il est, accroît considérablement les possibilités d'expérimentation sur $D$. pini et peut servir à la multiplication des parasites.

Diprion pini - Pinus silvestris - élevage - diapause - éclairage

Summary - A continuous rearing of Diprion pini L. in laboratory. A continuous rearing programme for Diprion pini $L$. has been developed in the laboratory from larvae collected in 2 localities (Sologne and Rambouillet forest near Paris. Insects have been reared here for more than 10 generations. The single non-diapausing insects have been used to get consecutive offspring. Corresponding larvae have been kept in photoperiod and under temperature conditions suitable for preventing diapause.

The larvae have been exposed to increased photophases and temperatures : $15 \mathrm{~h}$ at $16{ }^{\circ} \mathrm{C}$ up to the 3rd instar. Followed by $16 \mathrm{~h} 30$ at $20^{\circ} \mathrm{C}$. Egg laying and larval development took place in a single pine, without human manipulation. Larvae were subsequently reared on freshly cut twigs, which were renewed every 2-3 days. Several containers or boxes and 2 types of fluorescent lights were used.

Important variations in diapause rates were observed from 1 generation to the other, but the effect of biological insect processes or rearing conditions cannot be clearly defined. However, changes in lighting types appeared to affect the diapause rates. Fluorescent "true-lite" tubes, with a spectrum very similar to solar light and which are inducive to spawing, appear in particular to increase the number of diapausing insects. In any case, a high rate of immediate emergences (without diapause) has been observed where insects were continuously reared under these conditions over a period of more than 5 generations.

An important increase in sex-ratio has been observed in this continuous rearing process, which can be explained by several causes, but in particular, by consanguinity.

The above-mentioned rearing technique increases the possibilities of laboratory and field experiments on D. pini, and may be used for rearing parasites under artificial conditions.

Diprion pini - Pinus silvestris - rearing - diapause - lighting 


\section{INTRODUCTION}

Diprion pini L. hyménoptère Diprionidae, commet d'importants dégâts, au cours de gradations brutales et spectaculaires, dans toute l'Europe. II peut, durant ces gradations, défeuiller les pins sylvestres dans des forêts entières.

La biologie de cet insecte ayant déjà fait l'objet de nombreux articles (notamment Eichhorn 1976, 1977, 1978, 1979, 1982, Dusaussoy \& Geri 1966, Geri 1986, 1988, Geri \& Goussard 1988, 1989 ) on rappelera seulement quelques points essentiels :

- L'insecte est bivoltin dans le Bassin parisien.

- Les femelles pondent à l'extrémité des rameaux de pin sylvestre. En l'absence de fécondation elles ne produisent que des mâles.

- Les larves, groupées en colonies, consomment les aiguilles puis se transforment en éonymphes qui tissent un cocon.

- 11 y a 6 stades larvaires chez les individus femelles, 5 chez les mâles.

- Les éonymphes peuvent subir une diapause qui retarde les sorties d'adultes de quelques mois à plusieurs années.

- Cette diapause, dont le déterminisme est complexe, dépend d'un certain nombre de facteurs écologiques, en particulier de la photophase et de la température.

Les observations faites dans le Bassin parisien (Dusaussoy \& Geri 1966, 1971, Geri \& Dusaussoy 1966, Geri \& Goussard 1984, Geri $1986,1988)$, ont montré que les gradations se développent très rapidement. Des petites populations très peu parasitées et faiblement diapausantes, disposant d'un feuillage intact, se multiplient en quelques générations. Dès que la phase de culmination est atteinte, la raréfaction de la nourriture et de très nombreux insectes entomophages causent une forte mortalité des diprions. De nombreux individus entrent alors en diapause de longue durée. La phase de régression à lieu l'année suivante. Ces phénomènes s'amplifient : l'insecte subit, en outre, les conséquences d'une modification de la qualité de la nourriture, les aiguilles de l'année précédente ayant été en majorité consommées et le nouveau feuillage étant nocif pour les larves (Geri et al., $1985,1987,1988)$. Des réinfestations, généralement de faible importance, peuvent avoir lieu 1 à 2 ans après, à partir des individus en diapause, puis les insectes deviennent très rares.

Des phénomènes identiques ont été observés par Eichhorn (1983) dans le Palatinat et un processus très semblable a été décrit par Juutinen \& Varama (1986) pour un autre Diprionidae défoliateur du pin sylvestre Neodiprion sertifer Geoffr.
Cette évolution des gradations de $D$. pini semble donc très générale en Europe. Elle conduit à la situation suivante :

- Les parasites, l'alimentation et la diapause sont des facteurs écologiques, d'une très grande importance pour la dynamique des populations de $D$. pini.

- Il est presque impossible d'expérimenter de façon continue sur l'action de ces facteurs, à cause de la courte durée des gradations et de la rareté des populations naturelles durant les longues phases d'intergradation.

- L'élevage de l'insecte présente de grandes difficultés du fait même du phénomène de diapause qui s'oppose à la continuité des générations. A notre connaissance il n'a été obtenu sur un grand nombre de générations que par Michels (1983) avec des apports réguliers d'insectes prélevés dans la nature.

D'où la nécessité pour le chercheur de mettre au point un élevage permanent de Diprion. Par élevage permanent, nous entendons l'élevage d'insectes prélevés à l'origine dans des populations naturelles, de génération en génération, sans interruption par l'entrée en diapause et sans limitation du nombre de générations.

Cet élevage présenterait en outre l'intérêt :

- de permettre des élevages de parasitoïdes à des fins de lutte biologique (Geri et al., 1986),

- de disposer d'insectes élevés à l'abri des parasites pour les expérimentations,

- d'accroître les possibilités expérimentales en augmentant le nombre de générations annuelles, - de réduire la variabilité expérimentale due à la diversité des conditions du milieu naturel et à l'hétérogénéité génétique des populations,

- de permettre de tester certaines hypothèses concernant la diapause, notamment la nécessité d'un retour à l'état de diapause après plusieurs générations sans arrêt de développement, hypothèse envisagée pour expliquer les forts taux de diapause observés à la fin des gradations (Geri \& Goussard 1988).

Cet article fait le bilan des travaux réalisés depuis 1980 par I'INRA au centre de recherches d'Orléans pour mettre au point un tel élevage.

\section{MATÉRIELS ET MÉTHODES}

\section{Premiers essais avant 1986}

Les insectes destinés à l'élevage sont prélevés dans les forêts voisines d'Orléans (Sologne, forêts d'Orléans, de Rambouillet, de Fontainebleau) ou dans des populations originaires de ces forêts élevées en pépi- 
nière. Ils sont choisis dans des populations issues d'insectes diapausants, populations elles-mêmes généralement peu diapausantes. L'origine des insectes placés en élevage de 1982 à 1986 est présentée dans le Tableau !.

En se basant sur le fait que la diapause peut généralement être évitée en exposant les larves âgées à des photophases longues (Eichhorn 1976, 1977, 1978, 1979, Geri et al. 1984, Geri \& Goussard 1988), les premiers essais d'élevage ont été effectués en soumettant les insectes durant tout leur développement à une température élevée et à une photophase de 16h. A partir de 1982, après avoir constaté que les réactions des larves âgées dépendaient des conditions de photophase subies par les jeunes larves, on a utilisé des jours courts pour les jeunes larves et des jours longs dans des conditions de température plus éle-

Tableau I. Premiers essais d'élevage permanent de $D$. pini. Effectifs de cocons et pourcentages de sorties immédiates d'adultes observés à chaque génération. A. Souche constituée à l'automne 1982 à partir de larves prélevées en Sologne (Romorantin); élevage à $20^{\circ} \mathrm{C}, 12 \mathrm{~h}$ d'éclairement jusqu'au $3^{\mathrm{e}}$ stade, $16 \mathrm{~h}$ ensuite. Cocons ramenés à $12 \mathrm{~h}, 12 \mathrm{j}$ après leur formation; B. Souche constituée à l'automne 1983 à partir de larves prélevées en Sologne (Sennely); œufs à $12{ }^{\circ} \mathrm{C}$ $12 \mathrm{~h}$ d'éclairement; larves à $12{ }^{\circ} \mathrm{C}, 16 \mathrm{~h}$ jusqu'au $3^{\mathrm{e}}$ stade; $20^{\circ} \mathrm{C}, 16 \mathrm{~h}$ d'éclairement ensuite jusqu'à l'émergence des adultes; C. Souche constituée au printemps 1984 à partir d'adultes issus d'une population de la forêt d'Orléans de l'automne 1983. $12{ }^{\circ} \mathrm{C}, 12 \mathrm{~h}$ d'éclairement de l'œuf au $3^{\mathrm{e}}$ stade larvaire $\left(20^{\circ} \mathrm{C}, 12 \mathrm{~h}\right.$ pour la $1^{\text {re }}$ génération); $20^{\circ} \mathrm{C}, 16 \mathrm{~h}$ du $3^{\mathrm{e}}$ stade à la formation des cocons; $25^{\circ} \mathrm{C}, 16 \mathrm{~h}$ ensuite; $\mathrm{D}$. Souche constituée de la même façon. Elevage à $20^{\circ} \mathrm{C}, 17 \mathrm{~h} 30$ d'éclairement.

$N^{\circ}$

N. Coc

IM

A

1

2

3

4

B

1

2

3

C

1

2

3

4

D

1

2

3

No : numéro d'ordre de la génération; N. Coc. : effectif de cocons; IM : \% de sorties immédiates; * : forte mortalité des larves et des éonymphes. vées pour les larves âgées. Un essai a également été réalisé en 1984 avec une photophase très longue (17 h 30) constante.

Ces élevages ont été conduits dans des caissons en bois de $(2 \times 1 \times 1,50 \mathrm{~m})$ placés dans des pièces thermorégulées et éclairées par 3 tubes fluorescents "blanc industrie" assurant une intensité lumineuse de 5000 I au niveau des insectes (on appellera de façon résumée ces conditions "caisson B.I.»).

Chaque génération est obtenue à partir des adultes issus des individus non diapausants de la précédente. La ponte est obtenue en plaçant des couples de Diprion sur des petits pins sylvestres de moins d'un $m$, en godets, ensachés dans des bonnettes de toile. Après l'éclosion, les larves sont collectées sur ces pins au $2^{\mathrm{e}}$ stade larvaire et placées dans des cages d'élevage de $(60 \times 30 \times 30 \mathrm{~cm})$ sur des rameaux de pins sylvestres fraîchement coupés et immergés à leur base dans un pot rempli d'eau. Elles sont alimentées avec du feuillage frais tous les 2 ou $3 \mathrm{j}$ (Fig. 1C). Les cocons sont conservés dans de petites boîtes en plastique aérées (Fig. 1D).

A chaque génération l'effectif des cocons obtenus, la sex-ratio, la mortalité des éonymphes et le pourcentage de sorties immédiates sont notés. Des observations sont faites sans dénombrement sur la mortalité des larves.

\section{L'élevage à partir de 1986}

Deux souches d'élevage ont été constituées durant l'automne 1986 à partir d'insectes de la région de Sennely en Sologne (souche $A$ ), et à partir de diprions originaires de la forêt de Rambouillet élevés depuis 1984 en pépinière (souche $\mathrm{P}$ ).
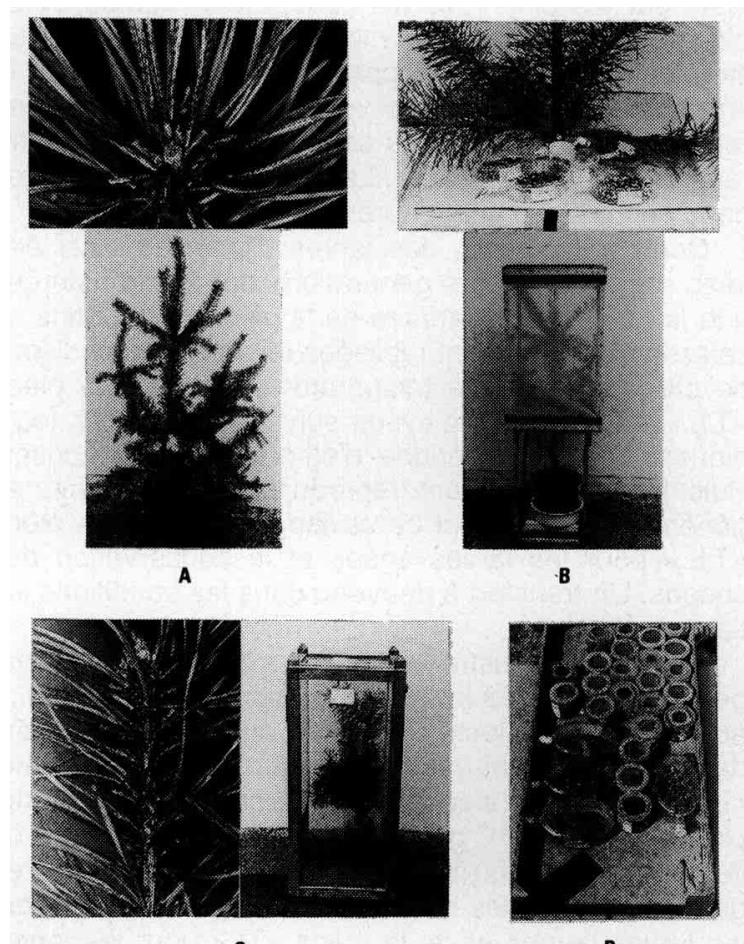

Fig. 1. Elevage de Diprion pini : A - Ponte, pin de ponte et d'élevage des jeunes larves; B - Conditionnement des cocons et des pins pour la ponte; $\mathrm{C}$ - Larves et cage d'élevage des larves âgées; D - Dispositif de conservation des cocons. 
Le principe général de l'élevage demeure inchangé, ainsi que les notations effectuées à chaque génération. Toutefois, afin de se rapprocher au maximum des conditions subies dans la nature, les insectes sont élevés à $16{ }^{\circ} \mathrm{C}$ en photophase $15 \mathrm{~h}$ durant les premiers stades, puis à $20^{\circ} \mathrm{C}$ en photophase $16 \mathrm{~h} 30$ à la fin de leur développement larvaire. En outre on utilise deux pièces thermorégulées de $(3 \times 2,40 \times 2,40 \mathrm{~m})$ équipées de 16 tubes fluorescents, les insectes recevant toujours $5000 \mathrm{I}$. La $1^{\text {re }}$ est éclairée en tubes "blanc industrie" (pièce “B.I.»); la seconde, réservée à l'élevage des larves âgées particulièrement sensibles à l'éclairement, est éclairée par des tubes «true-lite" (pièce "T.L.") dont le spectre lumineux est beaucoup plus proche de celui de la lumière solaire.

L'utilisation de ces pièces de grand volume permet d'obtenir la ponte des insectes sur des pins hauts de $2 \mathrm{~m}$, au feuillage relativement abondant qui constituent un bon substrat de ponte, et permettent d'élever l'insecte sans manipulation jusqu'au $3^{\ominus}$ stade larvaire (Fig. 1A). Le feuillage de ces pins d'au moins 5 ans semble proche du point de vue nutritionnel de celui des arbres de 10 à 15 ans servant à l'alimentation des larves âgées. Ces pins sont prélevés chaque année en pépinière à l'âge de 3 ans, mis en containers et cultivés durant 2 ans sur une aire d'élevage afin d'obtenir une production régulière de sujets en bon état et bien pourvus d'aiguilles.

Les deux pièces sont réglées de façon que la pièce "Bl" assure des conditions de photophase courte (15h) et de température basse $\left(16^{\circ} \mathrm{C}\right)$, tandis que la pièce "TL" assure des conditions de photophase longue (16h 30) et de température plus chaude $\left(20^{\circ} \mathrm{C}\right)$. La ponte a lieu dans la pièce "T.L.". Les cocons sont disposés au pied des pins, eux-mêmes enfermés dans des cages en treillis (Fig. 1B). Les adultes sortent des cocons et viennent pondre librement sur les pins. Puis les cages sont retirées et les pins sont transportés dans la pièce "B.I.». Après éclosion les larves se développent sur ces pins jusqu'à la fin du $3^{\ominus}$ stade. Elles sont alors transportées sur des rameaux coupés dans les cages d'élevage et transférées dans la pièce "T.L.". Les cocons sont conservés dans cette même pièce après leur formation.

Comparativement, des larves d'insectes ainsi élevées depuis plusieurs générations ont été transférées à la fin du $3^{\theta}$ stade larvaire de la pièce «B.I.» dans un caisson "B.I." où sont réalisées les mêmes conditions de photophase et de température que dans la pièce "T.L.". Elles y sont élevées suivant les mêmes techniques jusqu'à la période d'émergence des adultes. Puis les insectes sont reproduits de génération en génération en utilisant ce caisson au lieu de la pièce "T.L." pour les larves âgées et la conservation des cocons. Un transfert à nouveau dans les conditions initiales a été tenté.

La Figure 2 illustre le détail des conditions d'élevage des deux souches de génération en génération. A noter que les insectes prélevés au $4^{\mathrm{B}}$ stade larvaire dans les conditions naturelles ont d'abord été maintenus au $4^{e}$ stade larvaire à $20^{\circ} \mathrm{C}$, sous $16 \mathrm{~h}$ de photophase durant la $1^{r e}$ génération. Ce n'est qu'à partir de la $2^{\ominus}$ génération, qu'ils ont été élevés de génération en génération dans les conditions de la pièce «B.I." pour les larves jeunes et de la pièce “TL" pour les larves âgées. Sur la Fig. 2 les lettres $A$ et $P$ sont réservées aux insectes de ces deux souches maintenus dans ces conditions. Les autres lettres correspondent à des changements de conditions. Les chiffres comptabilisent les générations depuis le début de l'élevage.

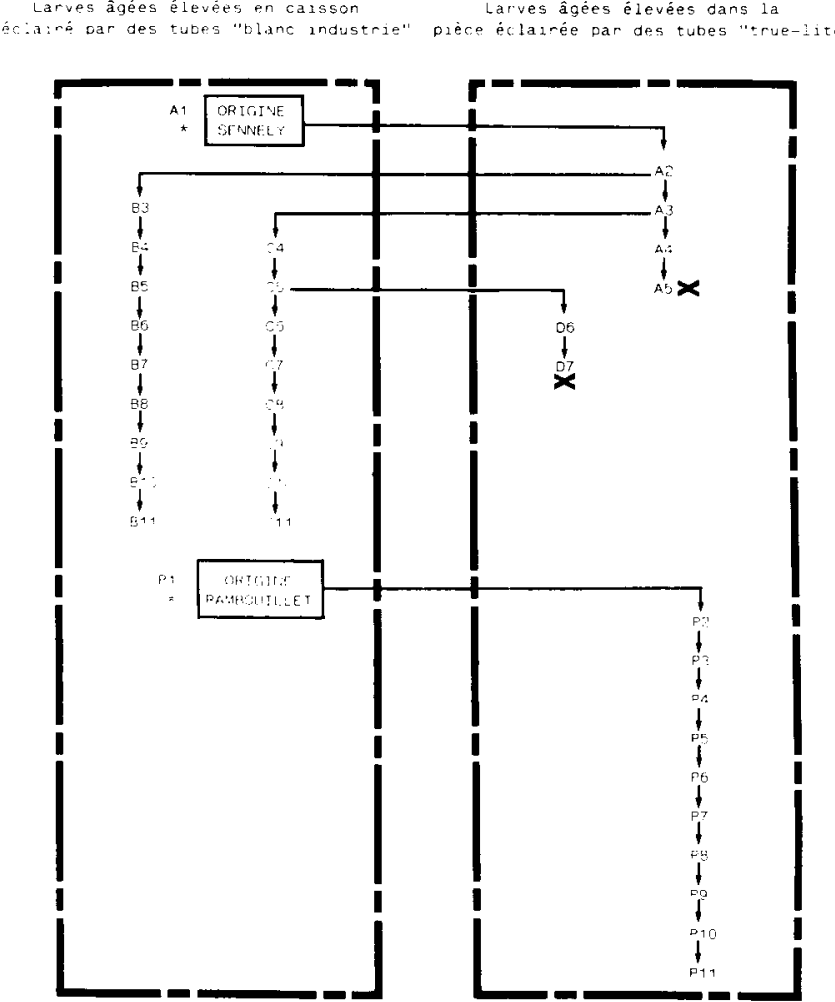

Fig. 2. Conditions d'élevage et filiation des populations des différentes souches de Diprion pini maintenues en élevage permanent au laboratoire. "Larves jeunes ayant évolué dans les conditions naturelles (durée du jour 12-13h). Larves âgées élevées en photophase $16 \mathrm{~h}$ à $20^{\circ} \mathrm{C}$. Dans tous les autres cas, les larves jeunes sont élevées en photophase $15 \mathrm{~h}$ à $16^{\circ} \mathrm{C}$ et les larves âgées en photophase $16 \mathrm{~h} 30$ à $20^{\circ} \mathrm{C}$. $>$ Extinction de la population.

\section{RÉSULTATS}

\section{Avant 1986}

Les tous premiers essais effectués en photophase constante $16 \mathrm{~h}$ ont conduit à des taux de diapause importants dès la deuxième génération. Les résultats des expériences réalisées ensuite, de 1982 à 1985, sont présentés avec les différentes conditions de température et de photophase subies par les larves et les cocons dans le Tableau I. Dans tous les cas, les insectes conservent un taux élevé de sorties immédiates durant les premières générations. Mais par suite d'une très forte mortalité à la $3^{\mathrm{e}}$ génération, souvent liée à une réduction de la fécondité des insectes, à des défauts de fécondation ou à la réapparition de très forts taux de diapause à la $4^{\theta}$ génération (Geri \& Goussard, 1988), l'élevage ne peut être poursuivi au delà de la $3^{\theta}$ ou de la $4^{\mathrm{e}}$ génération.

Ces premiers résultats semblent confirmer les difficultés des diprions à évoluer, sans arrêt de développement, durant plus de 3 générations consécutives. Mais de nombreuses difficultés 
techniques rencontrées avaient pu intervenir dans l'extinction des souches: traumatisme des adultes au cours de leur éclosion dans des boîtes plastiques et de leur transport sur les pins, mortalité des jeunes larves très fragiles causée par les manipulations, insuccès dans l'obtention des pontes lié à la protérandrie et aux faibles effectifs, médiocrité des petits pins comme support de ponte et comme source alimentaire; ces pins sont en effet peu attaqués dans la nature et la composition chimique de leur feuillage diffère notablement de celle des arbres âgés (Buratti et al. 1987, 1988).

\section{A partir de 1986}

\section{Insectes provenant de Sennely}

Plus de $88 \%$ des diprions ont un développement immédiat durant la première génération d'élevage. Après leur transfert dans les conditions de la pièce "B.I.» pour les jeunes larves et de la pièce “T.L." pour les larves âgées, le pourcentage d'individus diapausants s'accroît à la seconde génération, puis de nouveau à la $3^{e}$ génération, où il atteint $92 \%$. L'état sanitaire des insectes est satisfaisant. Ceci se traduit par un accroissement régulier des effectifs de cocons obtenus. Toutefois, s'ils sont maintenus dans ces conditions (A), le taux de diapause continue à s'accroître à la $4^{e}$ génération. En outre, une importante mortalité des larves et une mortalité notable des éonymphes réduisent le nombre d'individus. La $5^{\mathrm{a}}$ génération n'est pas obtenue (Tableau II).

Les insectes de la même origine transférés, après une génération ou deux générations, dans les conditions du caisson «B.I.» pour les larves âgées ( $B$ et $C$ ), ont un taux de diapause réduit. $A$ l'exception d'une nouvelle augmentation à la $4^{\mathrm{e}}$ génération pour les insectes de $B$, il décroît ensuite régulièrement de génération en génération, atteint un minimum à la $6^{\ominus}$ ou à la $7^{\mathrm{e}}$ génération, puis se stabilise à moins de $50 \%$ pour $\mathrm{B}$ et à moins de $35 \%$ pour $C$.

Malgré les prélèvements d'insectes destinés aux expériences et une certaine mortalité larvaire essentiellement due à des dépôts d'œufs trop nombreux sur les pins de ponte, les deux souches demeurent vigoureuses et une $11^{\mathrm{e}}$ génération est en cours de développement.

Lorsque ces insectes sont replacés dans les conditions de pièce "T.L." pour les larves âgées (D) le taux de diapause et la mortalité augmentent à nouveau. La souche disparaît en 2 générations.

\section{Insectes provenant de Rambouillet}

Ces insectes présentent durant la première génération et après leur transfert dans les conditions de la pièce «B.I.» pour les jeunes larves et de la pièce «T.L." pour les larves âgées, une évolution des taux de diapause strictement identique à celle des insectes originaires de Sennely jusqu'à la $3^{e}$ génération. Mais, à la différence de celle de ces derniers, la $4^{e}$ génération est beaucoup moins diapausante et un pourcentage élevé d'individus diapausants n'est observé qu'à la $5^{\mathbf{\theta}}$ génération. Le pourcentage d'individus en diapause décroît ensuite de génération en génération, jusqu'à la $7^{\mathrm{e}}$ génération, des valeurs un peu plus élevées étant observées ensuite. L'élevage peut être poursuivi; toutefois, les taux de diapause demeurent un peu plus importants que dans les populations originaires de Sennely dont les larves âgées sont élevées dans les conditions du caisson «B.I." et plusieurs générations présentent une certaine mortalité larvaire.

\section{DISCUSSION}

L'obtention de plus de 10 générations successives montre que l'élevage permanent de $D$. pini est à présent réalisé. Les effectifs, souvent de plusieurs centaines de cocons obtenus à la fin de chaque génération, confirment que cet élevage s'effectue dans des conditions satisfaisantes; et ce, d'autant plus qu'un certain nombre de larves sont prélevées pour expérimentation et que le nombre d'ovipositions est souvent limité par l'espace et les moyens disponibles.

Un certain nombre de problèmes restent posés. Ils concernent en particulier la mortalité des larves, l'accroissement des taux de diapause et le déséquilibre de la sex-ratio.

La trop forte concentration des œufs sur les pins de ponte constitue une des principales causes de mortalité larvaire relativement aisée à éviter. Les variations de la qualité du feuillage au cours de l'année et d'un pin à l'autre contribuent également à cette mortalité. En outre, l'élevage paraît passer par une phase particulièrement délicate à la $3^{\mathrm{e}}$ génération.

L'accroissement du nombre d'insectes en diapause observé au cours des 3 premières générations peut avoir plusieurs causes :

- la modification des conditions de température et de photophase entre la $1^{\text {re }}$ génération et les générations suivantes. Cette cause n'explique pas l'augmentation du taux de diapause à la $3^{\theta}$ génération.

- l'éclairage par les tubes «true-lite». En effet, les insectes maintenus dans la pièce éclairée par 
Tableau II. Observations faites sur les différentes souches de génération en génération, au cours de l'élevage permanent. A. Insectes provenant de Sennely; B. Insectes provenant de Rambouillet.

\begin{tabular}{|c|c|c|c|c|c|}
\hline & $\begin{array}{c}\text { Effectifs } \\
\text { de concons }\end{array}$ & $\begin{array}{c}\text { Sorties } \\
\text { immédiates } \\
\text { en } \%\end{array}$ & $\begin{array}{c}\text { Mortalité } \\
\text { des larves }\end{array}$ & $\begin{array}{c}\text { Mortalité des } \\
\text { éonymphes } \\
\text { en \% }\end{array}$ & $\begin{array}{r}\text { Sex ratio } \\
\text { des cocons }\end{array}$ \\
\hline \multicolumn{6}{|l|}{ A } \\
\hline $\mathrm{A} 1$ & 100 & 88,51 & & 8,00 & 0,30 \\
\hline A2 & 951 & 20,85 & & 7,45 & 1,61 \\
\hline A3 & 1063 & 7,90 & & 10,62 & 3,23 \\
\hline A4 & 58 & 6,90 & + & 28,07 & 0,50 \\
\hline A5 & $\infty$ & & & & \\
\hline B3 & 487 & 60,78 & & 3,29 & 1,45 \\
\hline B4 & 422 & 25,35 & & 13,98 & 1,36 \\
\hline B5 & 893 & 30,35 & & 6,38 & 2,39 \\
\hline B6 & 467 & 68,56 & & 2,14 & 1,10 \\
\hline B7 & 269 & 79,36 & + & 18,96 & 16,63 \\
\hline B8 & 267 & 50,74 & & 9,36 & 4,81 \\
\hline B9 & 572 & 83,19 & & 1,22 & 9,77 \\
\hline $\mathrm{B} 10$ & 819 & 52,94 & & 8,67 & 3,72 \\
\hline $\mathrm{C} 4$ & 422 & 18,25 & & 15,12 & 0,91 \\
\hline C5 & 450 & 30,67 & & 6,00 & 1,30 \\
\hline C6 & 806 & 92,32 & & 3,10 & 5,09 \\
\hline C7 & 464 & 90,85 & + & 6,02 & 2,32 \\
\hline $\mathrm{C} 8$ & 1281 & 65,12 & & 7,34 & 10,97 \\
\hline C9 & 536 & 86,86 & + & 1,69 & 3,60 \\
\hline C10 & 375 & 74,86 & + & 7,73 & 10,39 \\
\hline D6 & 350 & 78,05 & + & 1,71 & 5,66 \\
\hline D7 & 8 & 71,43 & + & 12,50 & que des mâles \\
\hline \multicolumn{6}{|l|}{ B } \\
\hline P1 & 60 & 88,33 & & 0 & 0,36 \\
\hline P2 & 445 & 15,23 & & 4,04 & 16,25 \\
\hline P3 & 134 & 7,46 & + & 3,73 & 0,50 \\
\hline P4 & 261 & 55,56 & & 0,77 & 1,76 \\
\hline P5 & 1254 & 5,82 & & 3,75 & 1,35 \\
\hline P6 & 481 & 49,23 & + & 5,61 & 17,90 \\
\hline P7 & 51 & 75,51 & + & 21,57 & 2,18 \\
\hline P8 & 55 & 69,81 & & 3,64 & 2,67 \\
\hline P9 & 83 & 34,18 & + & 4,82 & 2,77 \\
\hline P10 & 35 & 58,82 & + & 2,85 & 16,50 \\
\hline
\end{tabular}

A1, A2... : générations des différentes souches + forte mortalité larvaire.

larves âgées éclairées par des tubes "true-lite".

$><$ Extinction de la population.

sex ratio : $\frac{\text { nombre de mâles }}{\text { nombre de femelles. }}$

ces tubes ont subi, contrairement à ce qui était escompté, une augmentation importante des taux de diapause, tandis que ceux qui étaient replacés sous éclairage "blanc industrie" sont devenus moins diapausants. Cette incidence des changements qualitatifs de la lumière a été montrée expérimentalement (Geri \& Goussard, 1989).
- une tendance naturelle des insectes à entrer en diapause lorsqu'ils se sont développés de façon continue durant plusieurs générations. Les faits correspondent bien à cette hypothèse, mais elle ne peut être confirmée par l'élevage, compte tenu des autres causes possibles d'accroissement du nombre d'individus diapausants. 
Après la $3^{\theta}$ génération, dans certains cas, le taux de diapause s'est accru avec une forte mortalité aboutissant à la disparition de la population élevée, dans d'autres cas, il a progressivement diminué, parfois après un renforcement momentané, pour se stabiliser ensuite à des valeurs assez faibles. Le premier cas est surtout observé pour les insectes dont les larves âgées ont été élevées dans la pièce "true-lite", bien que les insectes originaires de Rambouillet paraissent s'être mieux adaptés aux conditions de cette pièce que ceux originaires de Sennely. Compte tenu du nombre limité d'adultes pris dans la fraction non diapausante pour engendrer à chaque génération la génération suivante, il est vraisemblable que cette évolution des différentes populations s'est accompagnée d'un phénomène de sélection. Finalement, au bout d'un certain nombre de générations, tous les insectes présentent un pourcentage important de sorties immédiates, quelles que soient leur origine et la nature de l'éclairement.

Durant l'élevage, la sex-ratio est fortement modifiée en faveur des mâles. Cette prédominance des mâles n'empêche généralement pas la poursuite des élevages, mais elle contribue à la disparition des populations de faibles effectifs (Tableau II, Fig. 2). Cette modification de la sexratio a plusieurs causes :

- l'émergence des adultes mâles est plus précoce. Cette protérandrie tend à s'accroître dans les conditions du laboratoire (Geri et al., 1985). II en résulte des défauts de fécondation dont la conséquence est une forte proportion de mâles dans la génération fille.

- la mortalité larvaire, notamment en cas de mauvaises conditions alimentaires, affecte plus particulièrement les individus femelles. En outre celles-ci sont souvent plus nombreuses que les mâles dans la fraction diapausante (Geri et al., $1985,1988)$. II en résulte également un accroissement de la sex-ratio. Toutefois, dans ce cas comme dans le cas précédent, la forte proportion de mâles favorise la fécondation à la génération suivante, d'où les fluctuations observées de la sex-ratio.

- l'élevage conduit logiquement à une forte consanguinité des insectes. II est connu que chez les Hyménoptères cette consanguinité peut se traduire par l'apparition de mâles diploïdes et de gynandromorphes (Crozier, 1971; Whiting, 1927; Whiting, 1943; White, 1973). Un tel phénomène n'est pas exclu dans le cas de $D$. pini. En effet un certain nombre de gynandromorphes ont été obtenus au cours de l'élevage et des électrophorèses enzymatiques suggèrent l'existence de mâles diploïdes (L. Baudoin, comm. pers.).

\section{CONCLUSION}

Les techniques utilisées depuis 1986 permettent désormais l'élevage permanent de $D$. pini. Elles sont susceptibles d'être encore améliorées et simplifiées. Par exemple, si une pièce contenant des pins entiers est nécessaire à l'accouplement et à l'élevage des jeunes larves, les larves âgées, qui sont alimentées avec des rameaux coupés, peuvent très bien être élevées dans des unités plus petites. Les variations de la nature de l'éclairement et de la qualité du feuillage demeurent deux problèmes importants. Les lampes «true-lite" permettent d'obtenir facilement l'accouplement et la ponte (Geri \& Goussard, 1989), mais leur utilisation pour l'élevage des larves est plutôt décevante. De toute façon, il convient d'éviter toute modification de la qualité de l'éclairage au cours de leur développement. La variabilité de l'alimentation peut être réduite en utilisant le feuillage de pins appartenant à un même clone.

Tel qu'il est actuellement réalisé, l'élevage répond dans l'ensemble aux objectifs recherchés, mais il doit être amélioré pour certains objectifs spécifiques. Ainsi, la vérification de l'hypothèse de l'augmentation de l'aptitude des diprions à entrer en diapause, après plusieurs générations de développement continu, nécessite l'élevage des insectes dans des conditions strictement identiques, de photophase, de température, de qualité de la lumière, d'alimentation, et même, de densité des individus, dès la $1^{\text {re }}$ génération. Un suivi génétique des populations est également nécessaire afin de tenir compte des phénomènes de sélection.

D'ores et déjà, l'élevage peut permettre d'élever des parasites à des fins de lutte biologique, en simplifiant encore les techniques pour obtenir des productions massives d'entomophages. L'obtention d'un certain nombre d'individus diapausants n'est pas gênante car ils peuvent être utilisés pour la multiplication de parasites spécifiques des éonymphes (Geri et al., 1986).

Il est également satisfaisant, pour obtenir toute l'année du matériel expérimental exempt de parasites. Ceci a permis d'accroître considérablement le champ des investigations menées sur D. pini, de fournir des insectes à d'autres laboratoires, et de réduire la variabilité des résultats des expériences. La consanguinité constitue un problème mais elle peut être évitée par des apports périodiques de mâles sauvages, tout en étant conscient que ces apports réintroduisent un facteur de variabilité. 


\section{RÉFÉRENCES}

Buratti L., Allais J.P. \& Barbier M. (1987) The resin acids involved in the relations between scots pine and the sawfly Dipion pini L. I. The contents of resin acids in the needles. In : Mechanisms of woody plant defenses against insects (Mattson W.J., Levieux J. \& Bernard Dagan C. eds) Springer Verlag New York, $171 \cdot 187$

Buratti L., Allais J.P., Geri C. \& Barbier M. (1988) Diterpene resin acid evolution in Scots pine Pinus silvestris needles and the regulation of the european pine sawfly (Diprion pini L.) populations. Ann. Sci. Forest. (accepté pour publication)

Crozier R.M. (1971) Heterozygosity and sex determination in haplo-diploidy. Am. Nat. 105, 399-412

Dusaussoy G. \& Geri C. (1966) Etude d'une population de Diprion pini L. (Hym. Symphites) en forêt de Fontainebleau. I. - Données biologiques sur Diprion pini L. et sur les principaux parasites. Ann. Soc. Entomol. Fr. (NS), 2, 503-534

Dusaussoy G. \& Geri C. (1971) Etude des populations résiduelles de Diprion pini L. après la gradation de 1963-1964. Ann. Sci. For. 28, 297-322

Eichhorn O. (1976) Dauerzucht von Diprion pini L. (Hym. Diprionidae) in laboratorium unter Berücksichtigung der Fotoperiode. Anz. Schädlingskde Pflanzen Schutz. Umweltscthutz. 49, 38-41

Eichhorn O. (1977) Autökologische Untersuchungen an Populationen der gemeinen Kiefern-Buschkornblattwespe Diprion pini L. (Hym. Diprionidae) III Laborzuchten. Z. Angew. Entomol. 84, 264-282

Eichhorn O. (1979) Autökologische Untersuchungen an Populationen der gemeinen Kiefern-Buschkornblattwespe Diprion pini L. (Hym. Diprionidae). IV - Generations und Schlüpfwellenfolge. Z. Angew. Entomol. 88, 378-398

Eichhorn O. (1978) Zur prognose der Schlüpfwellen und Generationenfolge beider gemeinen KierfernBuschkornblattwespe Diprion pini L. (Hym. Diprionidae). Anz. Schädlingskde Pflanzen Schutz. Umweltschutz 51, 65-69

Eichhorn O. (1982) Untersuchungen zur ökologie der Gemeinen Kiefern - Buschkornblattwespe. Diprion pini L. (Hym. Diprionidae) - VII Populations dynamische Factoren. Z. Angew Entomol. 94, 271-300

Eichhorn O. (1983) Untersuchungen zur ökologie der Gemeinen Kiefern-Buschkornblattwespe. Diprion pini L. (Hym. Diprionidae) VIII Verlauf der Massenvermehrung bei Speyer (Pflaz) 1976-1978. Z. Angew. Entomol. 96, 291-303

Geri C. (1986) Facteurs influençant la dynamique des populations de Diprion pini L. dans le Bassin parisien. 18th IUFRO Congress. Lubjana, Proceeding division 2, 695-705

Geri C. (1988) The pine sawfly in Central France In : Forest insects Populations dynamics. Plenum Berryman 377-405
Geri C. \& Dusaussoy G. (1966) Etude d'une population de Diprion pini L. en forêt de Fontainebleau. II. Etablissement d'une table de mortalité. Ann. Soc. Entomol. Fr. (NS) 2, 535-548

Geri C. \& Goussard F. (1984) Evolution d'une nouvelle gradation du Lophyre du pin (Diprion pini L.) dans le sud du Bassin parisien. I. Développement de la gradation et relation avec les facteurs du milieu. Ann. Sci. For. 41, 375-404

Geri C. \& Goussard F. (1988) Incidence de la photophase et de la température sur la diapause de Diprion pini L. (Hym. Diprionidae). Z. Angew. Entomol. 106, 150-172

Geri C. \& Goussard F. (1989) Incidence de la qualité de la lumière sur le développement et la diapause de Diprion pini L. (Hym. Diprionidae) Z. Angew Entomol. 108, 99-101

Geri C., Goussard F. \& Liger A. (1984) Influence de la photopériode sur les arrêts de développement de Diprion pini L. (Hym. Diprionidae). C.R. Acad. Agric. Fr. 70, 245-251

Geri C., Allais J.P., Goussard F., Liger A. \& Yart A. (1985) - Incidence de l'âge du feuillage sur le développement de Diprion pini L. (Hym. Diprionidae). Conséquences sur l'évolution des populations. Acta Oecol. Oecol. Appl. 6, 349-365

Geri C., Goussard F. \& Reveillon M. (1986) Réflexion sur l'utilisation des auxiliaires entomophages contre Diprion pini L. (Hym. Diprionidae). Entomophaga 31, 27-38

Geri C., Buratti L., \& Allais J.P. (1987) The resin acids involved in the relation between Scots pine and the sawfly Diprion pini L. II. Correlations with the biology of Diprion pini L. In : Mechanisms of woody plant defenses against insects. (Mattson W.J., Levieux J. \& Bernard Dagan C. eds) Springer Verlag New York, 189-201

Geri C., Goussard F., Allais J.P. \& Buratti L. (1988) Incidence de l'alimentation sur le développement et la diapause de Diprion pini (Hym. Diprionidae). $Z$. Angew Entomol. 106, 451-464

Juutinen P. \& Varama M. (1986) Ruskean Mäntypistiäisen (Neodiprion sertifer) esiintylminen Suomessa vuosina 1966-1983. Folia For. 662, 1-39

Michels F.P. (1983) Untersuchungen zur biologischen bekämpfung der gewohnlichen Kiefern-Bushkornblattwespe Diprion Pini L. (Hym. Diprionidae), mit insectviren (Kreuzinfections und Adaptationsversuche). Dr. rer. hort. Univesität Hannover.

White M.J.D. (1973) Animal cytology and evolution. 3th edition Cambridge University Press. Londres, 961p

Whiting A.R. (1927) Genetic evidence of diploid males in Habrobracon. Biol. Bull. Woods Hole 53, 438-449

Whiting P.W. (1943) Multiple alleles in complementary sex determination of Habrobracon. Genetics 28, 365382 